\title{
Chiral Dirac superconductors: Second-order and boundary-obstructed topology
}

\author{
Apoorv Tiwari $\odot,{ }^{1,2}$ Ammar Jahin, ${ }^{3}$ and Yuxuan Wang ${ }^{3}$ \\ ${ }^{1}$ Department of Physics, University of Zurich, Winterthurerstrasse 190, 8057 Zurich, Switzerland \\ ${ }^{2}$ Condensed Matter Theory Group, Paul Scherrer Institute, CH-5232 Villigen PSI, Switzerland \\ ${ }^{3}$ Department of Physics, University of Florida, Gainesville, Florida 32601, USA
}

(Received 15 June 2020; revised 17 October 2020; accepted 20 October 2020; published 1 December 2020)

\begin{abstract}
We analyze the topological properties of a chiral $p+i p$ superconductor for a two-dimensional metal and semimetal with four Dirac points. Such a system has been proposed to realize second-order topological superconductivity and host corner Majorana modes. We show that with an additional $\mathrm{C}_{4}$ rotational symmetry, the system is in an intrinsic higher-order topological superconductor phase, and with a lower $\mathrm{C}_{2}$ symmetry, is in a boundary-obstructed topological superconductor phase. The boundary topological obstruction is protected by a bulk Wannier gap. However, we show that the well-known nested Wilson loop is in general unquantized despite the particle-hole symmetry, and thus fails as a topological invariant. Instead, we show that the higher-order topology and boundary-obstructed topology can be characterized using an alternative defect classification approach, in which the corners of a finite sample are treated as a defect of a space-filling Hamiltonian. We establish "Dirac $+(p+i p)$ " as a sufficient condition for second-order topological superconductivity.
\end{abstract}

DOI: 10.1103/PhysRevResearch.2.043300

\section{INTRODUCTION}

The study of topological phases of matter concerns itself with classifying ground states of gapped quantum systems and characterizing them via certain robust properties which remain insensitive to adiabatic deformations [1-4]. A crucial manifestation of topological phenomena is the bulk-boundary correspondence which predicts the existence of nontrivial (ingappable, degenerate, or long-range entangled) degrees of freedom on the boundary of a topological phase purely by analyzing the bulk properties. Paradigmatic examples of such phenomena are the appearance of an odd number of Dirac cones on the surface of the three-dimensional (3D) topological insulator [5], ingappable helical modes on the edge of the quantum spin Hall insulator [6,7], and chiral Majorana modes on the edge of the $p+i p$ topological superconductor [8], to name a few.

In the past years there have been a gamut of developments that have generalized the bulk-boundary correspondence to include more subtle phenomena. Two classes of generalizations known as higher-order topological phases and boundaryobstructed topological phases are of relevance to this work. Broadly speaking, phases that have a gapped bulk as well as gapped codimension-1 boundaries but necessarily support nontrivial degrees of freedom on higher-codimension boundaries are known as higher-order topological insulators and superconductors [9-41]. A system that supports nontrivial

Published by the American Physical Society under the terms of the Creative Commons Attribution 4.0 International license. Further distribution of this work must maintain attribution to the author(s) and the published article's title, journal citation, and DOI. states on codimension- $q$ corners belongs to a $q$ th-order topological phase. Yet another subclass of topological phases, the so-called "boundary-obstructed topological phases" [32,42], also host states localized on higher-codimension boundaries. However, such states are not protected by the bulk energy gap. Instead, as the name suggests, they are protected by the boundary energy gap (or relatedly the bulk Wannier band gap). In the literature, such a topological phase is also referred to as an "extrinsic" higher-order topological phase [15], in distinction with the "intrinsic" ones protected by a bulk energy gap. The two phases are closely related, and their connection has been studied in Ref. [42] in the context of topological insulators, in which spatial symmetries play an important role. In this work we focus on topological superconductors, in which, as we shall see, the role of spatial symmetries is rather different. Specifically, we study a class of two-dimensional (2D) superconductors that can be either second-order topological superconductors $\left(\mathrm{HOTSC}_{2}\right)$ or boundary-obstructed topological superconductors $\left(\mathrm{BOTSC}_{2}\right)$ depending on the discrete rotational symmetry of the system.

The topological properties of Bogoliubov-de Gennes (BdG) Hamiltonians in the weak-pairing limit can often be understood simply and efficiently in terms of the Fermi surface properties of the normal state Hamiltonian and are independent of the details of the electronic structure away from the Fermi surface. This is not unexpected since Cooper pairing is indeed dominant only in the neighborhood of the Fermi surface. In one dimension (1D), a gapless fermionic system with two Fermi points subject to $p$-wave pairing is a topological superconductor with Majorana zero modes at its ends [8]. Similarly, in 2D a system with a Fermi surface that encloses the $\Gamma$ point in the Brillouin zone, subject to chiral $p+i p$ pairing is a topological superconductor that hosts chiral Majorana modes at its edge [43-48]. Similar low-energy 
criteria have been proposed for time-reversal-invariant superconductors [49] in various dimensions. All of the above results share the remarkable feature that the topological bulk-boundary correspondence is completely contingent on the low-energy description of the normal state. Apart from contributing toward a clear theoretical understanding, such criteria are useful from the perspective of materials search. It is therefore highly desirable to formulate higher-order and boundary-obstructed topology in the context of superconductors in terms of similar low-energy criteria.

Toward this end, a low-energy criterion was proposed in Ref. [16] for a topological superconductor with corner Majorana modes, which stated that a $2 \mathrm{D} \mathrm{HOTSC}_{2}$ can be realized in a doped two-band Dirac semimetal with four Dirac points in the presence of finite-range attractive interactions. With a finite density of states, i.e., for a nonzero chemical potential $\mu$, the leading pairing instability is toward a $p+i p$ order. A "minimal model" of such a state is given by the following Hamiltonian (see also Ref. [50]):

$$
\begin{aligned}
H= & \int d \boldsymbol{k}\left\{c^{\dagger}(\boldsymbol{k})\left(t \cos k_{x} \sigma_{x}+t \cos k_{y} \sigma_{z}-\mu\right) c(\boldsymbol{k})\right. \\
& \left.+c^{\dagger}(\boldsymbol{k})\left(\Delta \sin k_{x}+i \Delta \sin k_{y}\right) c^{\dagger}(\boldsymbol{k})+\text { H.c }\right\},
\end{aligned}
$$

in which $c(\boldsymbol{k})$ is a two-component spinor field in the band basis, and the normal state has four Dirac points at $( \pm \pi / 2, \pm \pi / 2)$. It has been argued that the zero modes remain robust upon various small deformations.

While Ref. [16] demonstrated the existence of four Majorana zero modes at the corners of a finite sample, it remained to be elucidated what its topological classification is, and what topological invariant characterizes its nontrivial topology. One well-known topological invariant for second-order topological insulator, both intrinsic and boundary-obstructed (extrinsic), with mirror reflection symmetries is the nested Wilson loop [10,11], defined as the polarization of a Wannier band, a band formed by orthonormal filled states that are extended in one direction but exponentially localized in the orthogonal direction. In the presence of mirror reflection symmetries, the nested Wilson loop is quantized to be 0 or $\frac{1}{2}$, and the second-order topology is captured by two nested Wilson loops along the $x$ and $y$ directions. However, if $\mu \neq$ 0 , the Hamiltonian in Eq. (1) breaks the accidental mirror symmetry [16], and the nested Wilson loop is unquantized. Further attempts to quantize the nested Wilson loop using particle-hole symmetry are also unsuccessful as explained in the main text. Importantly, in sharp contrast with the case of regular Wilson loop (polarization), the particle-hole symmetry does not impose quantization conditions on nested Wilson loops. Consequently, we argue that the nested Wilson loop does not generally provide a topological invariant for $\mathrm{BdG}$ Hamiltonians without mirror symmetries.

We note that recently an exhaustive classification scheme of $\mathrm{BdG}$ Hamiltonians with additional spatial symmetries [51-53] has been developed based on organizing the BdG as well as normal state bands according to their symmetry eigenvalues at high-symmetry points in the Brillouin zone. Within this approach, we prove that our BdG, when augmented with a $\mathrm{C}_{4}$ rotation symmetry, realizes an intrinsic $\mathrm{HOTSC}_{2}$ phase. However, such an ancillary $\mathrm{C}_{4}$ symmetry is rather peculiar
TABLE I. A BdG superconductor with four Dirac points in the normal state Hamiltonian and an odd-parity pairing term [Eq. (2)] hosts Majorana zero-mode corner states. When enriched by spatial $\mathrm{C}_{4}$ rotation symmetry, such a model is a second-order topological superconductor $\left(\mathrm{HOTSC}_{2}\right.$ ). When the $\mathrm{C}_{4}$ symmetry is reduced to a $\mathrm{C}_{2}$ subgroup, the model continues to host Majorana zero modes as a boundary-obstructed topological superconductor. We contrast these cases with the analogous cases without particle-hole symmetry, i.e., by treating the BdG Hamiltonian as an insulator wherein the phase with and without $\mathrm{C}_{4}$ symmetry is a second-order topological insulator $\left(\mathrm{HOTI}_{2}\right)$ and a trivial insulator, respectively.

\begin{tabular}{lcc}
\hline \hline Model & With $_{4}$ & With $_{2}$ \\
\hline With PH & HOTSC $_{2} ;$ & BOTSC $_{2} ;$ \\
& corner Majorana & corner Majorana \\
Without PH & HOTI $_{2} ;$ & Trivial \\
& filling anomaly & no filling anomaly \\
\hline \hline
\end{tabular}

and artificial. In particular, its corresponding operator satisfies $\widehat{\mathrm{C}}_{4}^{4}=-1$, and its eigenvalues are half-integers. For a superconductor in $2 \mathrm{D}$, it is more natural to have twofold rotation symmetry than fourfold rotation symmetry, since the former relates $\boldsymbol{k}$ with $\boldsymbol{-} \boldsymbol{k}$ and is crucial to a Cooper instability. In the presence of $\mathrm{C}_{2}$ symmetry, we find that our system is trivial in terms of intrinsic higher-order topology. Therefore, this approach does not capture the BOTSC $_{2}$ phase in the absence of $\mathrm{C}_{4}$ symmetry.

In this paper, in addition to the $\boldsymbol{k}$-space bulk approach described above, we provide an alternative real-space boundary approach. We view corner states of a finite-size Hamiltonian as topological defects of a space-filling Hamiltonian. Since a topological defect, by definition, being only dependent on the topological winding number, is insensitive to the details of the band structure away from the Fermi level we can directly work with a low-energy description of the model. Further, since a topological defect is locally insensitive of the bulk rotational symmetry, this real-space approach naturally lends itself to analyzing and proving the "Dirac $+p+i p$ " low-energy criterion for both $\mathrm{HOTSC}_{2}$ 's and $\mathrm{BOTSC}_{2}$ 's. In order to establish this criterion, we carefully study a general BdG Hamiltonian with four Dirac points in the normal state subject to oddparity pairing. The Dirac points of the normal state can be protected by a chiral symmetry, or a product of time-reversal and inversion symmetry, but both these symmetries are broken in the superconducting state. For our purposes, we simply take the Dirac points as an input. In addition to particle-hole symmetry, such a model may have certain spatial symmetries. We show that depending on whether or not one imposes the additional $\mathrm{C}_{4}$ rotation symmetry on the model, the Majorana modes can be protected by either the bulk or the edge energy gap implying intrinsic higher-order or boundary-obstructed topology, respectively. The different cases with and without $\mathrm{C}_{4}$ symmetry are summarized in Table I. To emphasize the role of particle-hole symmetry of the BdG Hamiltonian, we list in Table I the topological classification of the system had we interpreted it as an insulator without particle-hole symmetry. 
The rest of the paper is organized as follows. In Sec. II, we introduce our model and describe its various symmetries. In Sec. III, we study our model, enriched by $\mathrm{C}_{4}$ rotational symmetry on a $\mathrm{C}_{4}$-symmetric open geometry and show that it hosts corner Majorana modes. We first review a wellestablished symmetry-based indicator approach in Sec. III A to establish the existence of corner Majoranas before moving onto a real-space approach in Sec. III B. In Sec. IV, we relax the rotation symmetry constraint and show that the model is nontrivial in the sense of boundary-obstructed topology. In Sec. V, we conclude with a summary and some further directions.

\section{MODEL}

Consider a generic Bogoliubov-de Gennes (BdG) Hamiltonian $H_{\mathrm{BdG}}=\int d \boldsymbol{k} \Psi^{\dagger}(\boldsymbol{k}) \mathcal{H}(\boldsymbol{k}) \Psi(\boldsymbol{k})$, in which $\Psi^{\dagger}(\boldsymbol{k}) \equiv$ $\left(c^{\dagger}(\boldsymbol{k}), c(-k)\right)$ and

$$
\mathcal{H}(\boldsymbol{k})=\sum_{i=1,2}\left[f_{i}(\boldsymbol{k}) \Gamma^{i}+g_{i}(\boldsymbol{k}) \Gamma^{i+2}\right]-\mu \Gamma^{34},
$$

where $f_{i}(\boldsymbol{k})$ and $g_{i}(\boldsymbol{k})$ are even and odd functions, respectively. We use the convention $\Gamma^{1}=X^{31}, \Gamma^{2}=X^{33}, \Gamma^{3}=X^{10}, \Gamma^{4}=$ $X^{20}$, and $\Gamma^{5}=\Gamma^{1} \Gamma^{2} \Gamma^{3} \Gamma^{4}$ where $X^{\mu \nu}:=\tau^{\mu} \otimes \sigma^{\nu}$, such that $\sigma^{\mu}$ acts within the subspace of the normal state bands while $\tau^{\nu}$ act on the Nambu space indices. The $f_{1,2}$ terms correspond to the normal state dispersion, with Dirac points at the common zeros of $f_{1,2}$. Constrained by periodicity and parity of $f_{1,2}$, the number of Dirac points are necessarily multiples of four. In this work, we assume that there are four such Dirac points at $\pm \boldsymbol{k}_{F}$ and $\pm \boldsymbol{k}_{F}^{\prime}$. The chemical potential term is proportional to $\Gamma^{34}$, where we use $\Gamma^{\mu v}:=-i \Gamma^{\mu} \Gamma^{\nu}$. The pairing terms $g_{1,2}$ are of odd parity, which in the simplest case corresponds to a $p+i p$ symmetry. It has been shown [16] that such a generic BdG Hamiltonian is the superconducting ground state of a two-band Dirac semimetal with a finite-range attractive interaction. For the rest of this paper, we will take Eq. (2) as input and analyze its topology.

By construction, the BdG Hamiltonian is particle-hole symmetric, such that

$$
\mathrm{P} \mathcal{H}(\boldsymbol{k}) \mathrm{P}^{-1}=-\mathcal{H}(-\boldsymbol{k}),
$$

where $\mathrm{P}=X^{10} \mathrm{~K}$ and $\mathrm{K}$ implements complex conjugation. Furthermore, if $\mu=0$, the model has an additional chiral or sublattice symmetry generated by $S=\Gamma^{5}$ such that

$$
\mathrm{SH}(\boldsymbol{k}) \mathrm{S}^{-1}=-\mathcal{H}(k) .
$$

Therefore, the model Eq. (2) with $\mu \neq 0$ belongs to the AZ class $\mathrm{D}$ while for $\mu=0$, it belongs to class BDI. To analyze its higher-order topology, it will be instructive to augment the model with an additional $\mathrm{C}_{4}$ rotation symmetry generated by

$$
\widehat{\mathrm{C}}_{4}=\frac{\Gamma^{15}+\Gamma^{52}}{\sqrt{2}} \exp \left\{-\frac{i \pi \Gamma^{34}}{4}\right\},
$$

where $\Gamma^{5}=\prod_{i=1}^{4} \Gamma^{i}$. The rotation symmetry acts on the $\Gamma$ matrices as

$$
\widehat{\mathrm{C}}_{4}:\left[\begin{array}{c}
\Gamma^{1} \\
\Gamma^{2} \\
\Gamma^{3} \\
\Gamma^{4} \\
\Gamma^{5}
\end{array}\right] \mapsto \widehat{\mathrm{C}}_{4}\left[\begin{array}{c}
\Gamma^{1} \\
\Gamma^{2} \\
\Gamma^{3} \\
\Gamma^{4} \\
\Gamma^{5}
\end{array}\right]\left(\widehat{\mathrm{C}}_{4}\right)^{\dagger}=\left[\begin{array}{c}
\Gamma^{2} \\
\Gamma^{1} \\
\Gamma^{4} \\
-\Gamma^{3} \\
-\Gamma^{5}
\end{array}\right] .
$$

The rotational symmetry defined in Eq. (5) corresponds to a double group representation as can be seen explicitly from the fact that $\widehat{\mathrm{C}}_{4}^{4}=-1$. Invariance of the Hamiltonian under such a rotational symmetry action further imposes the following constraints on the functions $f_{i}(\boldsymbol{k})$ and $g_{i}(\boldsymbol{k})$ in addition to the one imposed by particle-hole symmetry:

$$
\begin{aligned}
& f_{1,2}\left(\mathbf{C}_{4} \triangleright \boldsymbol{k}\right)=f_{2,1}(\boldsymbol{k}), \\
& g_{1,2}\left(\mathrm{C}_{4} \triangleright \boldsymbol{k}\right)= \pm g_{2,1}(\boldsymbol{k}),
\end{aligned}
$$

where $\mathrm{C}_{4} \triangleright \boldsymbol{k}:=\left(k_{y},-k_{x}\right)$ denotes the action of $\mathrm{C}_{4}$ rotation in momentum space. As we mentioned $f_{1,2}$ each have a contour of zeros that intersects at four isolated Dirac points; these four Dirac points are related by the $\mathrm{C}_{4}$ symmetry. A simple example of a Hamiltonian invariant under such a set of symmetries is precisely Eq. (1). In BdG form, we have

$$
\mathcal{H}(\boldsymbol{k})=\sum_{i=1,2}\left[t \cos \left(k_{i}\right) \Gamma^{i}+\Delta \sin \left(k_{i}\right) \Gamma^{i+2}\right]-\mu \Gamma^{34} .
$$

In Sec. IV, we will relax the $\mathrm{C}_{4}$ symmetry and analyze the fate of the second-order topology.

\section{SECOND-ORDER TOPOLOGY FROM THE BULK PROTECTED BY ROTATIONAL SYMMETRY}

In this section we analyze the $\mathrm{C}_{4}$-symmetric BdG Hamiltonian described in Eq. (2) and show that it is in a HOTSC 2 phase. We adopt two complementary approaches to diagnose the second-order topology in such systems. First, in Sec. III A, we use a symmetry-indicator-based approach [51-55] to show that when treated as a $\mathrm{C}_{4}$-symmetric band insulator, the model in Eq. (2) is in the obstructed atomic limit which exhibits a filling anomaly and therefore hosts corner states on four $\mathrm{C}_{4}$ related corners. Furthermore, particle-hole symmetry requires that the corner states are Majorana zero modes. The results and methodology in Sec. III A closely follow recent works [51-53,55-58]. In Sec. III B, we adopt a real-space approach to show that the $\mathrm{C}_{4}$ symmetry pins topological defects at the four corners of the aforementioned spatial geometry. Then, second-order topology can be demonstrated using an index theorem in conjunction with the fact that topological defects in Altland-Zirnbauer classes D and BDI host $\mathbb{Z}_{2}$ and $\mathbb{Z}$ classified Majorana zero modes [59].

\section{A. Momentum-space approach from symmetry indicators}

Let us consider our model in Eq. (2) as a band insulator and for the moment ignore particle-hole symmetry. The model has two occupied and two unoccupied bands. Our strategy is to first analyze the $\mathrm{C}_{4}$ eigenvalues of the occupied bands at the high-symmetry points, from which we deduce the positions of the Wannier centers that induce these bands in the "atomic 
limit." We note that a proper definition of the atomic limit for superconductors has also been addressed [52,53,60], but for our purposes we will simply treat Eq. (2) as an atomic insulator. It is known that the rotation symmetry indices can only determine the Chern number of the system mod four [61]. Our model with vanishing chemical potential has an additional chiral symmetry which ensures vanishing Chern number and therefore Wannier representability. Furthermore, for small enough chemical potential that does not close the gap, which is what we assume, the model remains Wannier representable.

Now that we know there are no Wannier obstructions, we can proceed in calculating the $\mathrm{C}_{4}$ indices. Since the little groups at $\Gamma, M$ points and $X, X^{\prime}$ points contain the $\mathrm{C}_{4}$ and $\mathrm{C}_{2}$-symmetry operators, respectively, the corresponding symmetry eigenvalues for the occupied bands are topological indices, in that they cannot be changed without a bulk gap closing. Further, we note that the $p+i p$ order parameter $g_{i}(\boldsymbol{k})$ vanishes at these high-symmetry points due to Eq. (7), which means the order parameter does not directly affect the eigenvalues at these points. However, this does not imply that these terms do not play any role in the topology of the system, they have two important effects: (i) they affect the form of the $\mathrm{C}_{4}$ operator, indeed these terms are responsible for the $C_{4}^{4}=-1$ property of the operator; and (ii) without these terms, the system is gapless which renders the eigenvalues of the $\mathrm{C}_{4,2}$ operators at the high-symmetry points meaningless. The $\mathrm{C}_{4}$ symmetry also imposes that $f_{1}(0,0)=$ $f_{2}(0,0)=: f_{\Gamma}, f_{1}(\pi, \pi)=f_{2}(\pi, \pi)=: f_{M}$, and $f_{1,2}(\pi, 0)=$ $f_{2,1}(0, \pi)=: f_{X, X^{\prime}}$. The Hamiltonian (2) takes the following form at the high-symmetry points:

$$
\begin{aligned}
& \mathcal{H}_{\Gamma, M}=f_{\Gamma, M}\left[\Gamma^{1}+\Gamma^{2}\right], \\
& \mathcal{H}_{X, X^{\prime}}=f_{X, X^{\prime}} \Gamma^{1}+f_{X^{\prime}, X} \Gamma^{2} .
\end{aligned}
$$

We list the eigenvales for the high-symmetry points in Table II, from which it can be seen that the eigenvalues only depend on $\operatorname{sgn}\left(f_{\Gamma}\right)$ and $\operatorname{sgn}\left(f_{M}\right)$. There are four possibilities, corresponding to distinct configurations of atomic orbitals from which the filled bands can be induced. These have been summarized in Table III. Notably, depending on whether $\operatorname{sgn}\left(f_{\Gamma}\right) \operatorname{sgn}\left(f_{M}\right)=+1$ or -1 , the bands can be induced from a pair of atomic orbitals localized at the Wyckoff position 1a or $1 \mathrm{~b}$, i.e., at $\boldsymbol{r}=(0,0)$ or $\left(\frac{1}{2}, \frac{1}{2}\right)$. These two cases correspond to an unobstructed and obstructed atomic limit, respectively, as we shall see below.

Next, we confirm that our model (2) is indeed in an obstructed atomic limit by showing that the condition

TABLE II. The eigenvalues of the $\mathrm{C}_{4}$ operators at the $\Gamma$ and $M$ points as well as the $\mathrm{C}_{2}$ eigenvalues at the $X$ and $X^{\prime}$ points in the Brillouin zone.

\begin{tabular}{lc}
\hline \hline $\boldsymbol{k}_{*}$ & $\mathrm{C}_{4,2}$ eigenvalues of $\mathcal{H}\left(k_{*}\right)$ \\
\hline$\Gamma=(0,0)$ & $\left\{-\operatorname{sgn}\left(f_{\Gamma}\right) e^{\frac{i \pi}{4}}, \operatorname{sgn}\left(f_{\Gamma}\right) e^{-\frac{i \pi}{4}}\right\}$ \\
$X^{\prime}=(0, \pi)$ & $\left\{e^{\frac{i \pi}{2}}, e^{-\frac{i \pi}{2}}\right\}$ \\
$X=(\pi, 0)$ & $\left\{e^{\frac{i \pi}{2}}, e^{\frac{-i \pi}{2}}\right\}$ \\
$M=(\pi, \pi)$ & $\left\{-\operatorname{sgn}\left(f_{M}\right) e^{\frac{i \pi}{4}}, \operatorname{sgn}\left(f_{M}\right) e^{-\frac{i \pi}{4}}\right\}$ \\
\hline \hline
\end{tabular}

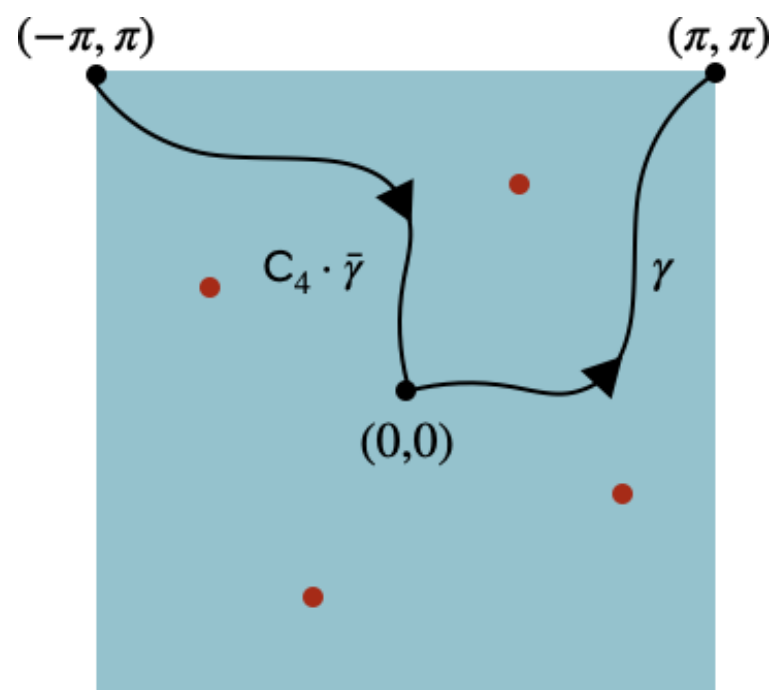

FIG. 1. A closed loop built from composing two $\mathrm{C}_{4}$ rotation related path segments $\gamma$ and $\mathrm{C}_{4} \cdot \gamma$ enclose a single Dirac point of the normal state Hamiltonian $\mathcal{H}_{\text {nor }}(\boldsymbol{k})$ [Eq. (10)].

$\operatorname{sgn}\left(f_{\Gamma}\right) \operatorname{sgn}\left(f_{M}\right)=-1$ follows from the fact that the normal state Hamiltonian in Eq. (2) contains a single Dirac cone per Brillouin zone quadrant. The normal state Hamiltonian takes the form

$$
\mathcal{H}_{\text {nor }}(\boldsymbol{k})=f_{1}(\boldsymbol{k}) \sigma^{x}+f_{2}(\boldsymbol{k}) \sigma^{z}=:\|f\| \hat{\boldsymbol{n}} \cdot \boldsymbol{\sigma},
$$

where $\|f(\boldsymbol{k})\|$ and $\hat{\boldsymbol{n}}$ are the norm and unit vector corresponding to $\boldsymbol{f}:=\left(f_{1}(\boldsymbol{k}), f_{2}(\boldsymbol{k})\right)$. The $\mathrm{C}_{4}$ transformation acts within the normal state via the operator

$$
\widehat{\mathrm{C}}_{4}^{\text {nor }}:=\frac{1}{\sqrt{2}}\left(\sigma^{x}+\sigma^{z}\right)
$$

as a mirror reflection about the $(1,1)$ axis in the $x$ - $z$ plane in spinor space, i.e., $\widehat{\mathrm{C}}_{4}^{\text {nor }}$, i.e., $\widehat{\mathrm{C}}_{4}^{\text {nor }}:\left(\sigma^{x}, \sigma^{z}\right) \rightarrow\left(\sigma^{z}, \sigma^{x}\right)$. The occupied state for the Hamiltonian (10), i.e., $|-\hat{\boldsymbol{n}}(\boldsymbol{k})\rangle$ therefore satisfies

$$
\begin{aligned}
\widehat{\mathrm{C}}_{4}^{\text {nor }}|\hat{\boldsymbol{n}}(\boldsymbol{k})\rangle & =e^{i \lambda}\left|\mathbf{M}_{(1,1)} \cdot \hat{\boldsymbol{n}}(k)\right\rangle \\
& =e^{i \lambda}\left|\hat{\boldsymbol{n}}\left(\mathbf{C}_{4} \cdot \boldsymbol{k}\right)\right\rangle,
\end{aligned}
$$

where $\mathrm{M}_{(1,1)}$ implements a reflection about the $(1,1)$ axis in spinor space. It follows that the spinor at rotationinvariant points $\Gamma$ and $M$ must correspond to $\hat{\boldsymbol{n}}\left(\boldsymbol{k}_{\Gamma, M}\right)=$ $\pm(1 / \sqrt{2}, 1 / \sqrt{2})$ which correspond to $\operatorname{sgn}\left(f_{\Gamma, M}\right)=\mp 1$. Now, consider a closed loop constructed from two $\mathrm{C}_{4}$-related paths $\gamma$ and $\mathbf{C}_{4} \cdot \bar{\gamma}$ as illustrated in Fig. 1. Such a loop encloses a

TABLE III. The signs of $f_{\Gamma}$ and $f_{M}$ completely determine the Wannier representation of the occupied bands. If $\operatorname{sgn}\left(f_{\Gamma}\right) \operatorname{sgn}\left(f_{M}\right)=$ -1 , the insulator is in an obstructed atomic limit.

\begin{tabular}{lcc}
\hline \hline $\operatorname{sgn}\left(f_{\Gamma}\right)$ & $\operatorname{sgn}\left(f_{M}\right)$ & Orbitals and Wyckoff position \\
\hline+ & + & $j=7 / 2, j=5 / 2 @ \boldsymbol{r}=(0,0)$ \\
+ & - & $j=7 / 2, j=5 / 2 @ \boldsymbol{r}=(1 / 2,1 / 2)$ \\
- & $j=3 / 2, j=1 / 2 @ \boldsymbol{r}=(1 / 2,1 / 2)$ \\
- & + & $j=3 / 2, j=1 / 2 @ \boldsymbol{r}=(0,0)$ \\
\hline \hline
\end{tabular}




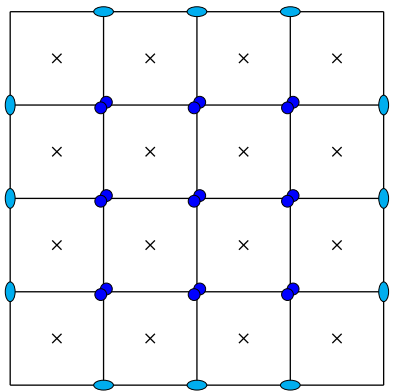

(a)

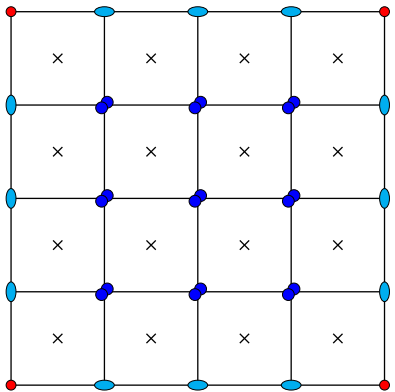

(b)
FIG. 2. Depiction of the Wannier centers for a sample with boundaries. The blue circles are bulk Wannier orbitals and there are two orbitals per unit cell both of which sit at the Wcykoff position $\boldsymbol{r}=\left(\frac{1}{2}, \frac{1}{2}\right)$. (The slight offsetting in the figure is just a visual aid to emphasize the existence of two centers.) The cyan ellipses are boundary orbitals and the red circles in (b) represent corner orbitals. When interpreted as an insulator system, filling the orbitals in a $\mathrm{C}_{4}$-symmetric manner necessarily leads to a violation of charge neutrality at the corners. In (a) and (b) we show the two different $\mathrm{C}_{4}$-symmetric fillings, the former have $-e / 2$ corner charge, while the latter have $e / 2$ corner charge. For a BdG Hamiltonian, this filling anomaly corresponds to corner Majorana zero modes.

single Dirac cone and therefore the spinor wave function must wind an odd number of times when traversing this closed path. Let us denote the winding number around a path $\gamma$ as $\mathrm{N}_{\mathrm{w}}(\gamma)$, then

$$
\mathrm{N}_{\mathrm{w}}\left(\gamma \circ\left(\mathrm{C}_{4} \cdot \bar{\gamma}\right)\right)=2 \mathrm{~N}_{\mathrm{w}}(\gamma),
$$

where $\circ$ denotes composition of paths. Therefore, we have $\mathrm{N}_{\mathrm{w}}(\gamma) \in \mathbb{Z}+1 / 2$. It can readily be seen that this can be achieved only if $\hat{\boldsymbol{n}}\left(\boldsymbol{k}_{\Gamma}\right)=-\hat{\boldsymbol{n}}\left(\boldsymbol{k}_{M}\right)$. This concludes the proof that $\operatorname{sgn}\left(f_{\Gamma}\right) \operatorname{sgn}\left(f_{M}\right)=-1$. We will be again using this relation in Sec. III B.

Interpreting the $\mathrm{BdG}$ Hamiltonian as a $\mathrm{C}_{4}$-symmetric insulator with open boundary conditions, it can be shown that for the phase with Wannier centers at $\left(\frac{1}{2}, \frac{1}{2}\right)$ there is no way to satisfy both $\mathrm{C}_{4}$ symmetry and neutrality. This phenomenon, known as the filling anomaly, necessitates corner states as long as the spatial rotation symmetry is unbroken [62]. It is important to check that the system has no polarization, otherwise the edges will be gapless and it would not make sense to talk about corner modes. That the polarization is zero can be checked by simple counting of the charges as shown in Fig. 2. Configuration (a) in Fig. 2 has the minimum amount of electrons to achieve neutrality of bulk and the edges, however, it is two electrons short for overall system neutrality. Since the system is $\mathrm{C}_{4}$ symmetric we will have $+e / 2$ charge localized on each corner. Two electrons cannot be added to the system in a $\mathrm{C}_{4}$-symmetric manner. The other closest configuration to neutrality is shown in Fig. 2(b). In this case, the system has two electrons in excess than that needed for neutrality, and hence $-e / 2$ charge localized on each quadrant.

In a noninteracting fermion system, a filling anomaly [27] manifests itself as a degeneracy of states, each localized at the opposite boundaries. In the presence of particle-hole symmetry, these degenerate localized states are pinned at zero energy. In the context of BdG Hamiltonians, these localized zero modes are Majorana zero modes. This is in contrast with systems with filling anomaly without particle-hole symmetry, in which the degenerate states are not zero modes and can even lie completely within the filled bands.

\section{B. Real-space approach from defect classification}

In this section we construct a real-space topological invariant to diagnose the purported Majorana corner states by treating the corner of a finite system as a topological defect of a space-filling Hamiltonian.

Consider placing the model in Eq. (2) on an open $\mathrm{C}_{4}$ symmetric spatial geometry $M$ such that the region outside $M$ corresponds to a BdG Hamiltonian with the same form as Eq. (2) but where the normal state, being featureless, has a vanishing Fermi surface. For concreteness, we may assume that the "outside" is described by a BdG Hamiltonian

$$
\mathcal{H}_{\text {triv }}(\boldsymbol{k})=\sum_{i=1,2}\left[-f_{0} \Gamma^{i}+\Delta \sin \left(k_{i}\right) \Gamma^{i+2}\right]-\mu \Gamma^{34},
$$

where $f_{0}>0$. Note that we have assumed a specific $p+i p$ form of the pairing potential for the sake of simplicity of presentation, however, the analysis would not change had we chosen general functions $g_{i}(\boldsymbol{k})$ that satisfy the symmetry constraints in Eq. (7). In going from inside to outside, the sign of $f_{i}$ must change at exactly one of the high-symmetry points $\Gamma$ or $M$ since $\operatorname{sgn}\left(f_{\Gamma}\right) \operatorname{sgn}\left(f_{M}\right)=-1$, as we proved earlier. Therefore, the boundary physics is determined entirely by the vicinity of that particular high-symmetry point. The Hamiltonians about the two high-symmetry points have the following Dirac-type forms:

$$
\begin{gathered}
\mathcal{H}\left(\boldsymbol{k}_{\Gamma}+\boldsymbol{q}\right)=\sum_{i}\left[f_{\Gamma} \Gamma^{i}+\Delta q_{i} \Gamma^{i+2}\right]-\mu \Gamma^{34}, \\
\mathcal{H}\left(\boldsymbol{k}_{M}+\boldsymbol{q}\right)=\sum_{i}\left[f_{M} \Gamma^{i}-\Delta q_{i} \Gamma^{i+2}\right]-\mu \Gamma^{34} .
\end{gathered}
$$

Both these Hamiltonians belong to class D or BDI depending on whether the chemical potential is vanishing or not as the chiral symmetry generated by $S=\Gamma^{5}$ is broken by the chemical potential term. It is known that topological defects in classes D and BDI host Majorana zero modes and are classified by $\mathbb{Z}_{2}$ and $\mathbb{Z}$, respectively $[59,63]$. Here, we first explicitly compute the invariant associated to a defect (localized at a given corner of $M$ ) using the chiral index theorem for the case of $\mu=0$. For the case of $\mu \neq 0$, it is known that the index is simply the chiral index evaluated modulo 2 as long as the chemical potential is small enough such that it does not close the gap, thereby potentially changing the topological invariant [64].

For concreteness let $f_{0}=f_{\Gamma}>0$ and $f_{M}<0$ in Eq. (15). Then, the low-energy physics is well described by the Dirac Hamiltonian linearized about the $\Gamma$ point. In order to show the existence of a Majorana zero mode on the edge, we consider a loop (see Fig. 3) $\ell$ parametrized by the angular variable $\theta$, which intersects the edge $\partial M$ at points $x_{0}$ and $x_{1}$ which are related by a $\mathrm{C}_{4}$ rotation. The mass of the Dirac Hamiltonian take values $f_{0}$ inside, $-f_{0}$ outside and vary smoothly in a narrow region close to the edges. For a general point in space there are two Dirac mass terms allowed: $\mathrm{m}_{1,2}(\boldsymbol{r}) \Gamma^{1,2}$. 


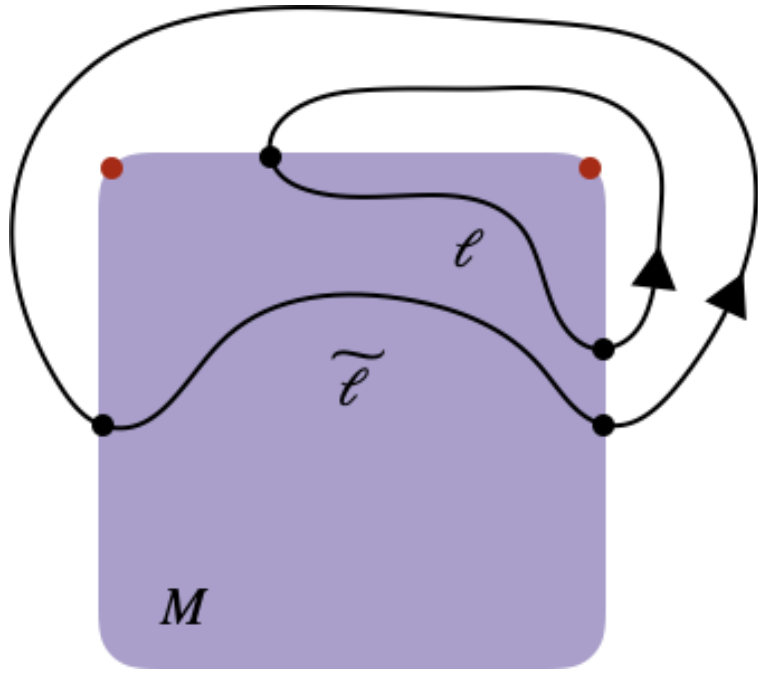

FIG. 3. A real-space sample with two different loops $\ell$ and $\tilde{\ell}$. $\ell$ encloses only one corner and intersects the boundary of the sample at $\mathrm{C}_{4}$-related points. $\tilde{\ell}$ encloses two corners and intersects the boundary of the sample at $\mathrm{C}_{2}$-related points.

Without loss of generality we assume that along the path $\ell$ the norm of the mass is constant, i.e., $\sqrt{\mathrm{m}_{1}^{2}+\mathrm{m}_{2}^{2}}=f_{0}:=\mathrm{m}$. We parametrize the masses by a single variable $\Phi(\theta)$ such that the defect Hamiltonian takes the form of a continuum Dirac model

$$
\mathcal{H}(\boldsymbol{q}, \theta)=\sum_{i} \Delta q_{i} \Gamma^{i+2}+\mathrm{m}\left[\cos (\Phi) \Gamma^{1}+\sin (\Phi) \Gamma^{2}\right] .
$$

Interestingly, we note that this defect Hamiltonian is dual to that for the celebrated Fu-Kane superconductor [65], which describes the $s$-wave pairing vortex of a Dirac fermion, while the first term comes from pairing and the second from normal state band structure in our case, it is the opposite in the FuKane superconductor. It is well known [59] that a pointlike mass defect in a class BDI Hamiltonian traps $\mathrm{N}_{\mathrm{w}}$ Majorana zero modes where $N_{w}$ corresponds to the winding number of the defect.

Consider a continuum model of the form $\mathcal{H}(\boldsymbol{q}, \theta)=$ $\sum_{i=1}^{4} \hat{n}_{i} \Gamma^{i}$ where $\hat{\boldsymbol{n}}$ is a map from $\mathbb{R}^{2} \times S^{1}$ to $S^{3}$. Such a Hamiltonian can be obtained from Eq. (16) by simply including an overall normalization factor of $\sqrt{\Delta^{2}|\boldsymbol{q}|^{2}+\mathrm{m}^{2}}$ which does not alter the winding number as it is positive definite. Furthermore, by adding a $\mathrm{C}_{4}$-symmetric $|\boldsymbol{k}| \rightarrow \infty$ regularization such as $\epsilon|\boldsymbol{k}|^{2}\left(\Gamma^{1}+\Gamma^{2}\right)$ and eventually taking the $\epsilon \rightarrow 0$ limit, the field $\boldsymbol{n}$ denotes a map from $S^{3}$ to $S^{3}$. The topological invariant then is the winding number of this map, which simplifies to the winding number of $\Phi$ :

$$
\begin{aligned}
\mathrm{N}_{\mathrm{w}}: & =\frac{1}{4 \pi^{2}} \int_{S^{3}} \epsilon^{i j k l} \boldsymbol{n}_{i} d \boldsymbol{n}_{j} \wedge d \boldsymbol{n}_{k} \wedge d \boldsymbol{n}_{l} \\
& =\frac{1}{2 \pi} \oint_{\ell} d \Phi .
\end{aligned}
$$

Let $\theta=0$ correspond to a point in the bulk and $\theta=\pi$ outside. The points $x_{0}, x_{1} \in \partial M$ correspond to $\theta=\alpha$ and $-\alpha$, respectively. Then, it can be seen that $\Phi(0)=\pi / 4$ while $\Phi(\pi)=$ $5 \pi / 4$. Finally, close to $x_{0}$ and $x_{1}, \Phi(-\alpha)=\pi / 2-\Phi(\alpha)$ due to the $\mathrm{C}_{4}$ action defined in Eq. (6). Using these relations it can be shown that

$$
\begin{aligned}
\mathrm{N}_{\mathrm{w}}(\gamma) & =\frac{1}{2 \pi} \oint_{0}^{2 \pi} d \Phi(\theta) \\
& =\frac{1}{2 \pi} \int_{0}^{\pi} d \Phi(\theta)+\frac{1}{2 \pi} \int_{\pi}^{0} d \Phi(-\theta) \\
& =\frac{1}{\pi} \int_{0}^{\pi} d \Phi(\theta) \\
& =(2 n+1),
\end{aligned}
$$

which signals that there are an odd number of zero modes localized on the edge between $x_{0}$ and $x_{1}$. For the case of finite chemical potential $\mu$, this index is reduced modulo 2 and, therefore, there is a single Majorana zero mode localized between $x_{0}$ and $x_{1}$. Similarly, we may consider the winding number around a loop $\tilde{\ell}$ that intersects $\partial M$ at two points $\tilde{x}_{0}$ and $\tilde{x}_{1}$ that are related by a $\mathrm{C}_{2}$ rotation. The symmetry constraint imposes that $\Phi(-\theta)=\frac{\pi}{2}-\left[\frac{\pi}{2}-\Phi(\theta)\right]=\Phi(\theta)$, therefore, the winding number corresponding to such a loop evaluates to

$$
\begin{aligned}
\mathrm{N}_{\mathrm{w}}(\tilde{\ell}) & =\frac{1}{2 \pi} \oint_{0}^{2 \pi} d \Phi(\theta) \\
& =\frac{1}{2 \pi} \int_{0}^{\pi} d \Phi(\theta)+\frac{1}{2 \pi} \int_{\pi}^{0} d \Phi(-\theta) \\
& =0 .
\end{aligned}
$$

Consequently, a path of the type $\tilde{\ell}$ encloses two Majorana zero modes with opposite topological indices.

\section{BOUNDARY-OBSTRUCTED TOPOLOGY IN THE ABSENCE OF $\widehat{\mathrm{C}}_{4}$}

In this section we focus on characterization of the topology of the chiral Dirac superconductor in the absence of $\mathrm{C}_{4}$ rotation symmetry. The Dirac points in the normal state are generically described by the following Hamiltonian:

$$
\mathcal{H}_{\text {nor }}(\boldsymbol{k})=f_{1}(\boldsymbol{k}) \sigma_{x}+f_{2}(\boldsymbol{k}) \sigma_{z},
$$

in which $f_{1,2}(\boldsymbol{k})$ are even functions and the location of the Dirac points is given by their simultaneous zeros. We assume as input that there are four Dirac points at $\pm\left(k_{x 0}, k_{y 0}\right)$ and $\pm\left(k_{x 0}^{\prime}, k_{y 0}^{\prime}\right)$. The Dirac points are locally (in $\boldsymbol{k}$ space) protected by a "minimal" symmetry which is the product of a timereversal $\mathrm{T}=\mathrm{K}$ symmetry and a $\widehat{\mathrm{C}}_{2}=\widehat{\mathrm{C}}_{4}^{2}$ rotation symmetry. For our purpose, we assume that the two symmetries are separately preserved, which makes Cooper pairing energetically favorable. In the presence of $p+i p$ pairing, the time-reversal symmetry is broken, and the only symmetry of the BdG Hamiltonian in consideration is a $\mathrm{C}_{2}$ rotational symmetry.

\section{A. Lack of bulk topological invariants}

We note that just like $\mathrm{C}_{4}$ symmetry, $\mathrm{C}_{2}$ symmetry can also potentially protect intrinsic higher-order topological phases. However, it can be confirmed by checking the symmetrybased indicators that our Hamiltonian is in the trivial phase as classified by $\mathrm{C}_{2}$ symmetry. To see this, note that the two 


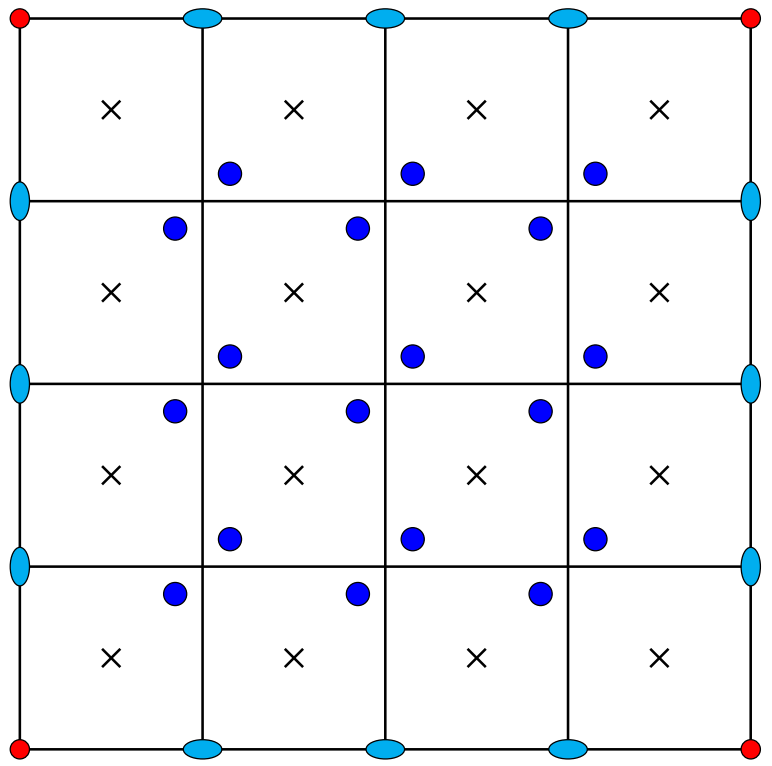

FIG. 4. A snapshot of the Wannier orbitals during a $\mathrm{C}_{2}$ symmetric process that connects the bulk Wannier centers from the Wyckoff positions $\boldsymbol{r}=(0,0)$ to $\boldsymbol{r}=\left(\frac{1}{2}, \frac{1}{2}\right)$. As argued in the main text, the edge orbitals are pinned to the $\boldsymbol{r}=\left(\frac{1}{2}, \frac{1}{2}\right)$ because of particle-hole symmetry and $\mathrm{C}_{2}$ symmetry. This shows the importance of particle-hole symmetry for the boundary-obstructed phase.

Wannier orbitals at $\boldsymbol{r}=\left(\frac{1}{2}, \frac{1}{2}\right)$ found in Sec. III can be adiabatically moved in opposite directions, and each can merge with a Wannier orbital from the neighboring Wannier orbital at the Wyckoff position $\boldsymbol{r}=(0,0)$, trivializing the phase without closing the bulk energy gap. Such a process is forbidden by the $\mathrm{C}_{4}$ symmetry but allowed by the $\mathrm{C}_{2}$ symmetry.

However, particle-hole symmetry ensures that the corner Majorana zero modes are stable unless there is a gap closing, in the bulk or on the boundary. We show that, in this case the second-order topology is extrinsic [15]. In our case without a topological invariant for the energy bands, the bulk energy gap does not need to close when the Majorana modes disappear. In this situation the Majorana zero modes can vanish via a gap closing at the edges only. In the terminology introduced in Ref. [42], the corner Majorana modes are protected by a boundary obstruction. Indeed, from the perspective of realspace Wannier orbitals, while in the bulk the two Wannier orbitals can continuously move from $\boldsymbol{r}=\left(\frac{1}{2}, \frac{1}{2}\right)$ to $\boldsymbol{r}=(0,0)$, at the boundary the sole filled Wannier orbital per unit cell cannot move. This is because in order to maintain zero polarization for the completely filled bands, the empty Wannier orbital [for convenience we refer to the negative (positive) energy states of the BdG Hamiltonian as filled (empty) bands] would have to move in the opposite direction, which then violates onsite particle-hole symmetry. We illustrate such a situation in Fig. 4.

A boundary-obstructed topological superconductor and a trivial superconductor with its edge wrapped by a 1D topological superconductor are similar in the sense that in both cases, nontrivial topology is hosted on a 1D subsystem. However, they can be distinguished by the fact that in the former case the edge topology ultimately comes from bulk properties. One such example is the quantized quadrupole insulator $[10,11,42]$ in which the fractional corner charge and the fractional edge polarization come from a fractional bulk quadrupole moment. As a result, an edge gap-closing transition is indeed captured by a bulk transition. However, such a transition happens in the bulk Wannier bands, rather than the energy bands. The Wannier band describes the spectrum, as a function of momentum along one direction, say $k_{x}$, of the projected position operator (also known as the Wannier Hamiltonian for a lattice system) $\hat{v}_{y}\left(k_{x}\right)$. Loosely speaking, $\hat{v}_{y}\left(k_{x}\right) \sim \hat{P}_{\text {occ }}(\boldsymbol{k}) \hat{y} \hat{P}_{\text {occ }}(\boldsymbol{k})$, where $\hat{y}$ is defined modulo 1 for a lattice system, and $\hat{P}_{\text {occ }}(\boldsymbol{k})$ is the projection operator onto the filled bands. More rigorously, according to the definition in Refs. [10,11],

$$
e^{2 \pi i \hat{v}_{y}\left(k_{x}\right)} \equiv \prod_{n=0}^{L_{y}-1} \hat{P}_{\mathrm{occ}}\left(k_{y}+\frac{2 \pi n}{L_{y}}, k_{x}\right),
$$

where $L_{y}$ is the system size along the $y$ direction. We note that the operator on the right-hand side of the above equation acts on a four-dimensional Hilbert space. However, because of the projection operators involved, it has a two-dimensional null space, and effectively the Wannier Hamiltonian is two dimensional. Further, $\hat{v}_{x}\left(k_{y}\right)$ is not single valued but defined modulo 1 . For definiteness we set $\left|\hat{v}_{x}\left(k_{y}\right)\right| \leqslant \frac{1}{2} \cdot{ }^{1}$

The eigenvalues $\left\{v_{x, \pm}\left(k_{y}\right)\right\}$ of the Wannier Hamiltonian correspond to the center of the hybrid Wannier states, localized in the $x$ direction and extended in the $y$ direction with momentum $k_{y}$. In our case, we find that the edge gap closing upon which the corner Majorana zero modes disappear is also detected by a bulk Wannier gap-closing transition. As a concrete example, we consider the following BdG Hamiltonian:

$$
\begin{aligned}
H= & \left(\cos k_{x}+\gamma_{x}\right) \sigma_{x} \tau_{z}+\cos k_{y} \sigma_{z} \tau_{z}-\mu \tau_{z} \\
& +\Delta \sin k_{x} \tau_{x}+\Delta \sin k_{y} \tau_{y},
\end{aligned}
$$

where normal state Dirac points and corner Majorana zero modes exist for $\left|\gamma_{x}\right|<1$ [see Fig. 5(b)]. At $\gamma_{x}=1$ there is a gap closing in the $x$-edge spectrum, through which the corner Majorana modes annihilate. At the same point, we show in Fig. 5(a) that there is a Wannier gap closing for the Wannier Hamiltonian $\hat{v}_{y}\left(k_{x}\right)$ at $k_{x}=0$, exactly where the edge gap closes.

One may ask if the bulk Wannier transition is captured by the change in a bulk topological invariant. In the presence of mirror symmetries, such a topological invariant is the Berry phase of the 1D Wannier band, known as the nested Wilson loop $[10,11,42]$, which is quantized by symmetry to take the value 0 or $\frac{1}{2}$. In our case, the $p+i p$ pairing order in general violates all mirror symmetries. One may naively expect that the particle-hole symmetry to quantize the nested Wilson loop. However, the two Wannier bands from the filled states with opposite eigenvalues of the Wannier Hamiltonian $\hat{v}_{y}\left(k_{x}\right)$ are not related by particle-hole symmetry; the two

\footnotetext{
${ }^{1}$ One subtlety is that the eigenstates of the Wannier Hamiltonian are not a Wannier state, but rather a Bloch state with definite $k_{x}$ and $k_{y}$. Its eigenvalues, on the other hand, are independent on $k_{x}$ and correspond to the center position of the Wannier states. We have suppressed the $k_{x}$ argument in the definition of $\hat{v}_{x}\left(k_{y}\right)$.
} 


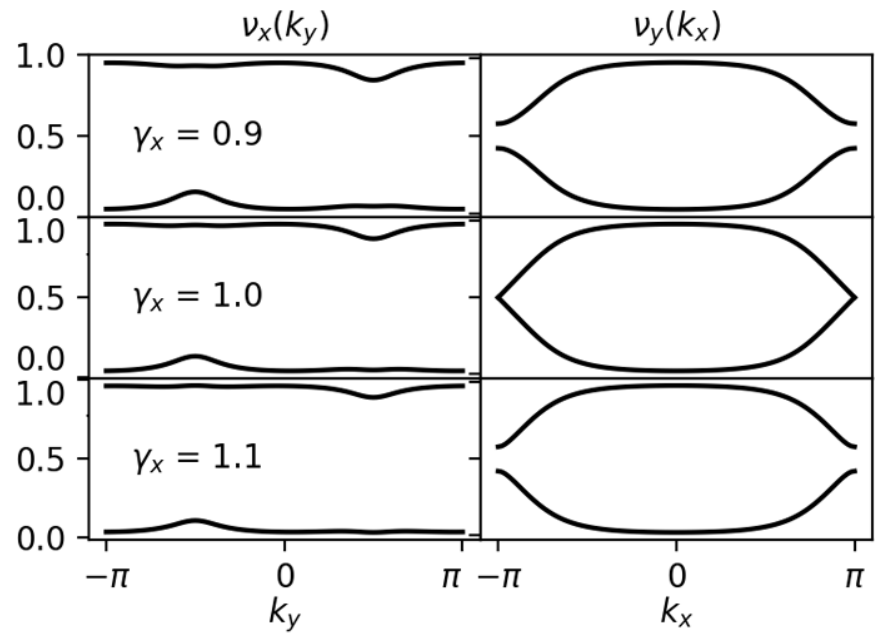

(a)

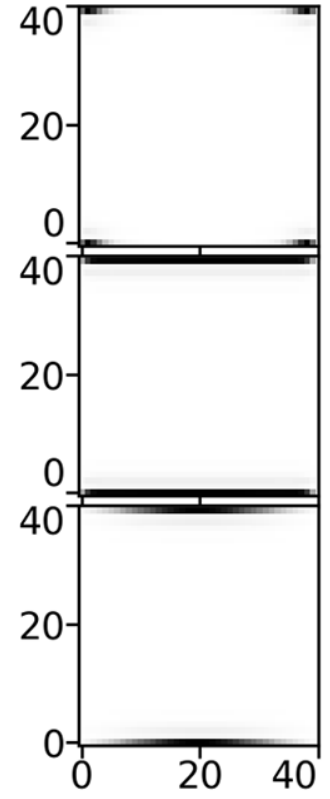

(b)

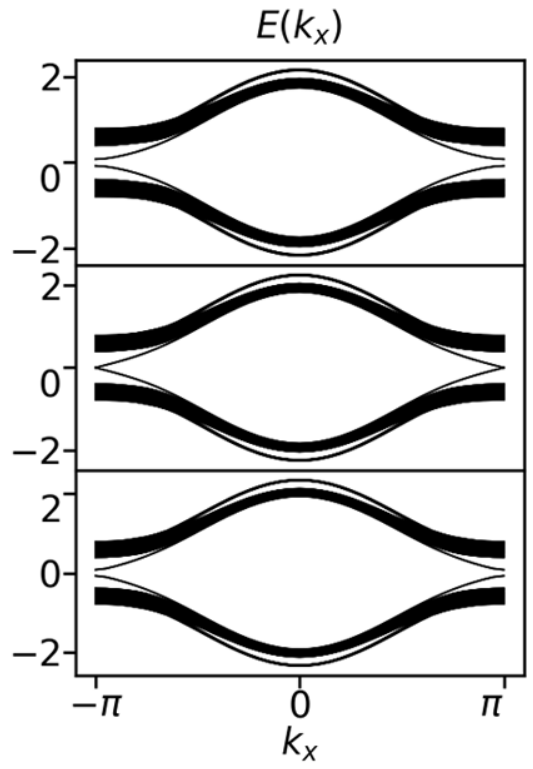

(c)

FIG. 5. Snapshots of the Wannier spectrum as $\gamma_{x}$ is changed. $\mu=0.2$ and $\Delta=0.4$ for all graphs. Top, middle, and bottom panels are for $\gamma_{x}=0.9,1$, and 1.1, respectively. (a) Shows the Wannier bands for Wilson loops along $x(y)$ on the left (right). For this graph, we changed our convention for the range of the Wannier bands to be $[0,1)$ to make the gap closing easier to visualize. In studying the system with open boundary conditions in both the $x$ and the $y$ directions with grid size $(40 \times 40)$, (b) shows the probability densities for the four wave functions with the lowest energies. (c) Shows the spectrum of the system in a cylindrical geometry, with periodic boundary condition along the $x$ direction. The size of the system along the $y$ direction is 40 . The graph shows the lowest 100 levels.

bands form a Hilbert subspace of the filled bands only, and particle-hole conjugation does not transform one to the other. Due to the $\mathrm{C}_{2}$ symmetry, the Wannier Hamiltonian satisfies $\hat{v}_{y}\left(k_{x}\right)=-\hat{\mathrm{C}}_{2} \hat{v}_{y}\left(-k_{x}\right) \hat{\mathrm{C}}_{2}$, which can be thought of as a composite of chiral symmetry and 1D inversion symmetry. However, unlike the mirror symmetry, this symmetry does not host any topologically nontrivial classifications.

To circumvent this difficulty with the Wannier Hamiltonian, one can consider the pair of Wannier bands from the filled state and the empty states with the same eigenvalue for $\hat{v}_{y}$ and $\hat{v}_{y}^{\prime} \sim \hat{P}_{\mathrm{emp}}(\boldsymbol{k}) \hat{y} \hat{P}_{\mathrm{emp}}(\boldsymbol{k})$, respectively $\left(\hat{P}_{\mathrm{emp}}=1-\hat{P}_{\mathrm{occ}}\right.$ is the projection operator to the empty bands). They are eigenstates of the following Wannier-projected Hamiltonian:

$$
\begin{aligned}
H_{P^{ \pm}}\left(k_{x}\right) & =P^{ \pm}\left(k_{x}\right) H P^{ \pm}\left(k_{x}\right), \text { where } \\
P^{ \pm}\left(k_{x}\right) & \equiv \frac{1 \pm \hat{P}_{\mathrm{occ}}(\boldsymbol{k}) \operatorname{sgn}\left(\hat{v}_{y}\right) \pm \hat{P}_{\mathrm{emp}}(\boldsymbol{k}) \operatorname{sgn}\left(\hat{v}_{y}^{\prime}\right)}{2} .
\end{aligned}
$$

The Wannier-projected Hamiltonian is not to be confused with the Wannier Hamiltonian, but it shares the same eigenstates with $v_{y}\left(k_{x}\right)\left[v_{y}^{\prime}\left(k_{x}\right)\right]$ for filled (empty) bands. When the Wannier gap closes, the eigenstates $\left|v_{y, \pm}\left(k_{x}\right)\right\rangle$ develop a singularity as a function of $k_{x}$, which can be detected both in $\hat{v}_{y}\left(k_{x}\right)$ and $H_{P^{ \pm}}\left(k_{x}\right)$.

In the Hilbert subspace subtended by these two bands, particle-hole conjugation indeed transforms one band to the other. However, since the projection operators $\hat{P}^{ \pm}$depend on $k_{y}$ and are thus nononsite in the $y$ direction, the projected particle-hole symmetry operator is also nononsite. Indeed, it is not difficult to show that the projected particle-hole operator for the Wannier projected Hamiltonian $H_{P^{ \pm}}\left(k_{x}\right)$ is given by

$$
\begin{aligned}
& \tilde{\mathrm{P}}\left(k_{x}\right) K=P^{ \pm}\left(k_{x}\right) \mathrm{P}\left[P^{ \pm}\left(-k_{x}\right)\right]^{*} K, \\
& H_{P^{ \pm}}\left(k_{x}\right)=-\tilde{\mathrm{P}}\left(k_{x}\right) H_{P^{ \pm}}^{*}\left(-k_{x}\right) \tilde{\mathrm{P}}^{\dagger}\left(k_{x}\right) .
\end{aligned}
$$

Such a nononsite operator does not quantize the Berry phase of the Wannier band, i.e., the nested Wilson loop, since in the proof of Berry phase quantization by the regular particle-hole symmetry, one needs to commute the particle-hole operator with $\partial_{k}$ [66]. Indeed, the nested Wilson loop can be computed via

$$
\begin{aligned}
e^{2 \pi i \hat{v}_{y}^{ \pm x}\left(k_{x}\right)} & \equiv \prod_{n=0}^{L_{y}-1} \hat{P}^{ \pm x}\left(k_{x}, k_{y}+\frac{2 \pi n}{L_{y}}\right), \\
\hat{P}^{ \pm x}(\boldsymbol{k}) & \equiv \frac{1 \pm \hat{P}_{\mathrm{occ}}(\boldsymbol{k}) \operatorname{sgn}\left(\hat{v}_{x}\right)}{2} .
\end{aligned}
$$

Similar as with the definition of the Wannier Hamiltonian, the nested Wilson loop operator as defined on the right-hand side of the above equation acts on a four-dimensional Hilbert space, but it has a three-dimensional null space. This makes the nested Wilson loop a $c$ number. A direct evaluation of the nested Wilson loop $\boldsymbol{P}^{v}(\boldsymbol{k})=\left(v_{x}^{+y}\left(k_{y}\right), v_{y}^{+x}\left(k_{x}\right)\right)$ shows that they are not only unquantized, but also depend on their respective starting point $k_{y, x}$. For the Hamiltonian (22) at $\Delta=0.4$, and $\mu=0.2$, gives $\boldsymbol{P}^{v}\left(\frac{\pi}{2}, \frac{\pi}{2}\right)=(0.46,0.5)$ for $\gamma_{x}=0.9$ and $\boldsymbol{P}^{v}\left(\frac{\pi}{2}, \frac{\pi}{2}\right)=(0.96,0.5)$ for $\gamma_{x}=1.1$.

Still, the bulk Wannier transition corresponds to the change in a topological invariant. With the projected particle-hole symmetry $\tilde{\mathrm{P}}\left(k_{x}\right)$, the Wannier transition occurs through a gap closing at high-symmetry momenta, e.g., $k_{x}=0, \pi$. The 


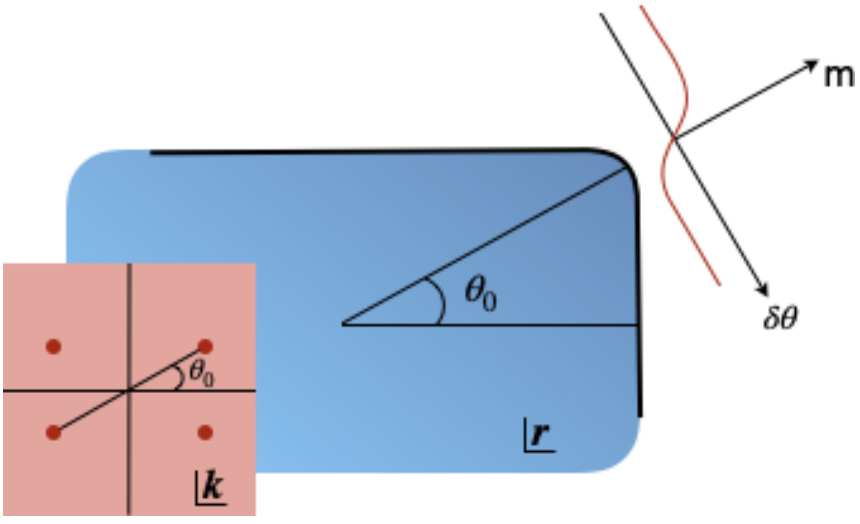

FIG. 6. An illustration of the defect Hamiltonian in Eq. (27). We first restrict to the line joining two Dirac points related by $\mathrm{C}_{2}$ symmetry. Then, within perturbation theory, it can be shown that the edge traps a pointlike mass defect where the $\theta_{0}$ line intersects the edge of the sample in real space.

Wannier-projected Hamiltonian $H_{P^{ \pm}}\left(k_{x}=0, \pi\right)$, as two zerodimensional subsystems, are invariant under the respective projected particle-hole symmetry $\tilde{\mathrm{P}}\left(k_{x}=0, \pi\right) \mathrm{K}$ and do each admit a $\mathbb{Z}_{2}$ classification $[1,2,66]$. The Wannier transition, through which the corner Majorana modes annihilate, is thus captured by the change in one of the $\mathbb{Z}_{2}$ invariants of the Wannier-projected Hamiltonian. However, since the particlehole symmetry $H_{P^{ \pm}}$at $k_{x}=0$ and $\pi$ are different, we cannot use their $\mathbb{Z}_{2}$ invariants to construct a "relative parity" that captures the property of the whole band $\left\{\left|v_{y, \pm}\left(k_{x}\right)\right\rangle\right\}$. As a result, these $\mathbb{Z}_{2}$ invariants do not capture the presence or absence of corner Majorana modes: it only detects the change in their existence. In the next section we directly show the existence of the corner Majorana modes from low-energy properties of the model through the defect classification approach.

\section{B. Real-space approach from defect classification}

We consider a system with rounded corners, whose size is much greater than the lattice scale, as shown in Fig. 6. Without loss of generality, we take the BdG Hamiltonian

$$
\begin{aligned}
H(\boldsymbol{k})= & f_{1}(\boldsymbol{k}) \sigma_{x} \tau_{z}+f_{2}(\boldsymbol{k}) \sigma_{z} \tau_{z}-\mu \tau_{z} \\
& +\epsilon g_{1}(\boldsymbol{k}) \tau_{x}+\epsilon g_{2}(\boldsymbol{k}) \tau_{y} .
\end{aligned}
$$

By the twofold rotation symmetry $\widehat{\mathrm{C}}_{2}=\widehat{\mathrm{C}}_{4}^{2}=\tau_{z}, f_{1,2}(\boldsymbol{k})$ and $g_{1,2}(\boldsymbol{k})$ are even and odd functions of $\boldsymbol{k}$, respectively. For convenience we take the weak-pairing limit where $\epsilon$ is small; increasing $\epsilon$ will not change our result. In the absence of additional spatial symmetries, we define the corner direction to be pointing from $\boldsymbol{k}=0$ to a pair of Dirac points, say at $\pm k_{F}\left(\cos \theta_{0}, \sin \theta_{0}\right)$. (Without loss of generality, we assume a rectangular lattice.) The rounded corner is parametrized by an angle $\theta \in(0, \pi / 2) \ni \theta_{0}$. We define the local momentum coordinates $\left(k_{\|}(\theta), k_{\perp}(\theta)\right)$ as locally parallel and perpendicular to the piece of edge parametrized by $\theta$. In turn, one can express $k_{x}$ and $k_{y}$ in terms of the local $k$ coordinates and $\theta$ as

$$
\begin{aligned}
& k_{x}(\theta)=k_{\perp} \cos \theta-k_{\|} \sin \theta, \\
& k_{y}(\theta)=k_{\perp} \sin \theta+k_{\|} \cos \theta .
\end{aligned}
$$

Let us focus on the slice of the bulk attached to the piece of edge at $\theta_{0}$. Since our goal is to obtain the low-energy modes on a smooth edge, we take a continuum limit and expand around small $k_{\|}$. We expand the Hamiltonian using the local $k$ coordinates at $\theta=\theta_{0}$. Before we do so, it is convenient to perform a unitary transformation on the Hamiltonian $H \rightarrow \tilde{H}=U H U^{\dagger}$, $\sigma_{x, z} \rightarrow \tilde{\sigma}_{x, z}\left(k_{\perp}\left(\theta_{0}\right)\right)=U\left(k_{\perp}\left(\theta_{0}\right)\right) \sigma_{x, z} U^{\dagger}\left(k_{\perp}\left(\theta_{0}\right)\right), \quad \tau_{x, y} \rightarrow$ $\tilde{\tau}_{x, y}\left(k_{\perp}\left(\theta_{0}\right)\right)=U\left(k_{\perp}\left(\theta_{0}\right)\right) \tau_{x, y} U^{\dagger}\left(k_{\perp}\left(\theta_{0}\right)\right)$, where

$$
\begin{aligned}
U\left(k_{\perp}\left(\theta_{0}\right)\right)= & \exp \left(\frac{i \sigma_{y}}{2} \tan ^{-1} \frac{f_{2}\left(k_{\perp}, k_{\|}=0\right)}{f_{1}\left(k_{\perp}, k_{\|}=0\right)}\right) \\
& \times\left.\exp \left(-\frac{i \tau_{z}}{2} \tan ^{-1} \frac{g_{2}\left(k_{\perp}, k_{\|}=0\right)}{g_{1}\left(k_{\perp}, k_{\|}=0\right)}\right)\right|_{\theta_{0}} .
\end{aligned}
$$

This transformation is not onsite (since its Fourier transform is not a $\delta$ function), but since it is periodic and smooth in $k_{\perp}$ $\left(\tan ^{-1}\left[f_{2}(\boldsymbol{k}) / f_{1}(\boldsymbol{k})\right]\right.$ is smooth even at the Dirac point), it is exponentially localized in real space. Thus, such a transformation does not change the exponential localization of wave packets. After the transformation, the Hamiltonian takes a simple form at $k_{\|}=0$ :

$$
\tilde{H}\left(k_{\perp}, k_{\|}=0 ; \theta_{0}\right)=\tilde{f}_{1}\left(k_{\perp}\right) \sigma_{x} \tau_{z}+\epsilon \tilde{g}_{1}\left(k_{\perp}\right) \tau_{x}-\mu \tau_{z},
$$

where

$$
\begin{aligned}
& \tilde{f}_{1}\left(k_{\perp}\right)=f_{1}\left(k_{\perp}, k_{\|}=0\right) \sqrt{1+\frac{f_{2}^{2}\left(k_{\perp}, k_{\|}=0\right)}{f_{1}^{2}\left(k_{\perp}, k_{\|}=0\right)}}, \\
& \tilde{g}_{1}\left(k_{\perp}\right)=g_{1}\left(k_{\perp}, k_{\|}=0\right) \sqrt{1+\frac{g_{2}^{2}\left(k_{\perp}, k_{\|}=0\right)}{g_{1}^{2}\left(k_{\perp}, k_{\|}=0\right)}} .
\end{aligned}
$$

The Hamiltonian (29) describes two decoupled copies of 1D $p$-wave superconductor. As long as $\mu$ does not exceed the bandwidth, they are in the weak-pairing phase with Fermi momenta at the two Dirac points. They give rise to two zero modes at the boundary, which, in a disk geometry, is the edge with well defined $k_{\|}$. The edge zero-mode wave function can be found via solving a differential equation with the replacement $k_{\perp} \rightarrow i \partial_{\perp}$ with the proper boundary conditions. This has been done in Ref. [67]. Following the results there, in the weak-pairing limit, the wave function comes predominantly from the low-energy components in the bulk, i.e., modes near the Fermi point. The $k$-space part of the wave function of the edge state is given by

$$
\begin{aligned}
\psi\left(k_{\perp}\right) & =\mathcal{N} \int_{0}^{\infty} e^{i k_{\perp} x} \sin \left(k_{F} x\right) e^{-\Delta x} \frac{d x}{2 \pi} \\
& =\frac{\mathcal{N}}{4 \pi}\left[\frac{1}{k_{\perp}+k_{F}+i \Delta}-\frac{1}{k_{\perp}-k_{F}+i \Delta}\right],
\end{aligned}
$$

where $\Delta=\epsilon \gamma_{1}\left(k_{F}\right)$ and $\mathcal{N}$ is a normalization factor. In the weak-pairing limit, $\left|\psi\left(k_{\perp}\right)\right|^{2}$ indeed is strongly peaked at $\left|k-k_{F}\right| \lesssim \Delta$. The internal part of the wave function is an eigenstate of $\tilde{\sigma}_{x} \tilde{\tau}_{y}$. We can further expand the Hamiltonian for small $k_{\|}$as

$$
\begin{aligned}
\tilde{H}\left(k_{\|}, k_{\perp} ; \theta_{0}\right)= & \tilde{f}_{1}\left(k_{\perp}\right) \sigma_{x} \tau_{z}+\epsilon \tilde{g}_{1}\left(k_{\perp}\right) \tau_{x}-\mu \tau_{z} \\
& +\varphi_{1}\left(k_{\perp}\right) k_{\|} \sigma_{x} \tau_{z}+\varphi_{2}\left(k_{\perp}\right) k_{\|} \sigma_{z} \tau_{z} \\
& +\epsilon \gamma_{1}\left(k_{\perp}\right) k_{\|} \tau_{x}+\epsilon \gamma_{2}\left(k_{\perp}\right) k_{\|} \tau_{y} .
\end{aligned}
$$


Since $f_{1,2}(\boldsymbol{k})$ are even functions and $g_{1,2}(\boldsymbol{k})$ are odd, one can vary that the expansion coefficients $\varphi_{1,2}\left(k_{\perp}\right)$ are an odd function and $\gamma_{1,2}\left(k_{\|}\right)$are even. For small $k_{\|}$, one can solve the spectrum of $\tilde{H}$ by perturbation theory. For a small $k_{\|}$, the spectrum of (32) can be found by perturbation theory

$$
\begin{aligned}
h\left(k_{\|}, \theta_{0}\right) & =\int d k_{\perp}\left|\psi\left(k_{\perp}\right)\right|^{2} \hat{P}_{\perp}^{+} H\left(k_{\|}, k_{\perp} ; \theta_{0}\right) \hat{P}_{\perp}^{+} \\
& =\epsilon \gamma_{2}\left(k_{F}\right) k_{\|} P_{\perp}^{+} \tau_{y},
\end{aligned}
$$

where $P_{\perp}^{ \pm}=\left(1 \pm \sigma_{x} \tau_{y}\right) / 2$ is the internal projection operator for the two edge states. We have used the fact that $P_{\perp}^{+} \tau_{y} P_{\perp}^{+}=$ $P_{\perp}^{+} \tau_{y}$. Importantly, we see that only the $\gamma_{2}\left(k_{\perp}\right)$ term survives the projection: $\varphi_{1,2}$ terms drop out because they are odd in $k_{\perp}$, and $\gamma_{1}$ term gets projected out by $P_{\perp}^{+}$.

We also expand the edge Hamiltonian as a function of coordinate $\delta \theta=\theta-\theta_{0}$, which we treat as a small quantity. For this edge, the parallel and perpendicular momenta related to those at $\theta_{0}$ via

$$
\begin{aligned}
& k_{\|}(\theta)=k_{\|}\left(\theta_{0}\right)-k_{\perp}\left(\theta_{0}\right) \delta \theta, \\
& k_{\perp}(\theta)=k_{\perp}\left(\theta_{0}\right)+k_{\|}\left(\theta_{0}\right) \delta \theta .
\end{aligned}
$$

At $k_{\|}(\theta)=0$, we have, to leading order, $k_{\|}\left(\theta_{0}\right)=k_{\perp}\left(\theta_{0}\right) \delta \theta$ and $k_{\perp}\left(\theta_{0}\right)=k_{\perp}(\theta)$. The Hamiltonian (32) can then be reexpressed with local $\boldsymbol{k}$ coordinates at $\theta$ as

$$
\begin{aligned}
\tilde{H}\left(k_{\|}=0, k_{\perp} ; \theta\right)= & \tilde{f}_{1}\left(k_{\perp}\right) \sigma_{x} \tau_{z}+\epsilon \tilde{g}_{1}\left(k_{\perp}\right) \tau_{x}-\mu \tau_{z} \\
& +\varphi_{1}\left(k_{\perp}\right) k_{\perp} \delta \theta \sigma_{x} \tau_{z}+\varphi_{2}\left(k_{\perp}\right) k_{\perp} \delta \theta \sigma_{z} \tau_{z} \\
& +\epsilon \gamma_{1}\left(k_{\perp}\right) k_{\perp} \delta \theta \tau_{x}+\epsilon \gamma_{2}\left(k_{\perp}\right) k_{\perp} \delta \theta \tau_{y} .
\end{aligned}
$$

Without the terms $\propto \delta \theta$, this again describes two decoupled 1D topological superconductors, leading to edge zero modes. The $\delta \theta$ terms can be treated perturbatively. We have

$$
\begin{aligned}
h\left(k_{\|}=0, \theta_{0}+\delta \theta\right) & =\int d k_{\perp}\left|\psi\left(k_{\perp}\right)\right|^{2} \hat{P}_{\perp}^{+} H\left(k_{\|}, k_{\perp} ; \theta_{0}\right) \hat{P}_{\perp}^{+} \\
& =\varphi_{2}\left(k_{F}\right) k_{F} \delta \theta P_{\perp}^{+} \sigma_{z} \tau_{z} .
\end{aligned}
$$

Now we see that only the $\varphi_{2}\left(k_{\perp}\right) k_{\perp}$ term survives the momentum and internal projection since this term is now even in $k_{\perp}$. Combining Eqs. (33) and (36), we get the edge Hamiltonian near $\theta_{0}$ and with small $k_{\|}^{\prime}$ :

$$
h\left(k_{\|}, \theta_{0}+\delta \theta\right)=\epsilon \gamma_{2}\left(k_{F}\right) k_{\|}\left(P_{\perp}^{+} \tau_{y}\right)+\varphi_{2}\left(k_{F}\right) k_{F} \delta \theta\left(P_{\perp}^{+} \sigma_{z} \tau_{z}\right) .
$$

Since $P_{\perp}^{+}$commutes with both $\tau_{y}$ and $\sigma_{z} \tau_{z}$, we have $\left\{P_{\perp}^{+} \tau_{y}, P_{\perp}^{+} \sigma_{z} \tau_{z}\right\}=0$. As $P_{\perp}^{+}$projects the internal Hilbert space dimension from 4 to 2 , we can represent the edge Hamiltonian in the projected Hilbert space as

$$
h\left(k_{\|}, \theta_{0}+\delta \theta\right)=\alpha k_{\|} s_{x}+\beta \delta \theta s_{y},
$$

where $\alpha=\epsilon \gamma_{2}\left(k_{F}\right)$ and $\beta=k_{F} \varphi_{2}\left(k_{F}\right)$, and $s_{x, y}$ are Pauli matrices.

From a classification perspective, for a 1D system the $\mathbb{Z}_{2}$ defect classification (in class D) is very simple, whose topological index is simply the relative sign of the mass term on the two sides of $\theta=\theta_{0}$. From (38), it is nontrivial in our case. This completes our proof; notice that even though the nontrivial topology comes from an edge Hamiltonian, it is ultimately determined by the bulk properties. By analogy with the 1D Jackiw-Rebbi model [68], the Hamiltonian in (38) hosts a Majorana zero mode localized at $\theta=\theta_{0}$.

It is instructive to consider the localization length of a rounded corner with radius $R, k_{\|}=-i \partial_{\theta} / R$. By solving Eq. (38), we found that the angular localization of the zero mode is given by

$$
\xi_{\theta}=\sqrt{\frac{\beta}{\alpha R}} \sim \sqrt{\frac{\epsilon a_{0}}{R}},
$$

where $a_{0} \sim 1 / k_{F}$ is the lattice constant. On the other hand, for the localization length, we have

$$
\xi \sim \sqrt{\epsilon R a_{0}},
$$

which vanishes in the sharp edge limit $R \rightarrow 0$ and weakpairing limit $\epsilon \ll 1$, and diverges as in the straight edge limit $R \rightarrow \infty$. The straight edge thus hosts delocalized gapless states; this is not surprising since this edge corresponds to a single angle with $\theta=\theta_{0}$.

Two remarks are in order here. First, it is possible to analyze the defect classification using a 2D space-filling "bulk" Hamiltonian, for which one would proceed almost identically as in the $\mathrm{C}_{4}$-symmetric case. However, here there is no symmetry protecting the mass pattern described in Sec. III B. It is thus possible to alter the topological index by deforming the 2D Hamiltonian and destroy the corner zero mode. What we found in this section, however, is that such a gap-closing deformation cannot occur as long as the bulk Dirac points in the normal state persist.

Second, it is clear that the above analysis does not require the normal state band structure have exactly four Dirac points. It instead applies to any two Dirac points from the two bands with $E_{ \pm}(\boldsymbol{k})= \pm \sqrt{f_{1}^{2}(\boldsymbol{k})+f_{2}^{2}(\boldsymbol{k})}$. One caveat, however, is that $f_{1,2}(\boldsymbol{k})$ are required to be even functions and, consequently, the two Dirac points at opposite momenta to have the same chirality. One notable candidate is the two Dirac points in the flat bands of twisted bilayer graphene which have the same chirality owing to fragile topology [69]. We leave this interesting extension to future work.

\section{SUMMARY}

In this work, we analyzed the second-order topology of a generic two-band doped Dirac semimetal in 2D with four Dirac nodes that are subject to $p+i p$ pairing. We showed that this model realizes either a HOTSC $\mathrm{H}_{2}$ or a BOTSC 2 phase, depending on the presence of an additional $\mathrm{C}_{4}$ symmetry. The two topological superconducting phases are intimately connected, with the difference being whether the energy gap protecting the Majorana modes is from the bulk or the boundary. We showed that while the nested Wilson loop approach in general fails to capture the boundary topological obstruction, both intrinsic higher-order topology and boundary-obstructed topology are naturally captured in an alternative defect classification approach. We thus establish the "Dirac $+(p+$ ip)" as a low-energy criterion for $\mathrm{TSC}_{2}$ phase, which can 
be viewed as an extension of the family tree of $p$-wave TSC's.

Based on our results, it would be interesting to search for chiral superconductivity in Dirac materials, such as the surface of topological crystalline insulators and graphene-based systems. Moreover, our result can be generalized to three dimensions to $p$-wave superconductivity in Weyl semimetals, which we leave to future work.

\section{ACKNOWLEDGMENTS}

We thank T. Bzdusek, M. Lin, T. Hughes, and T. Neupert for useful discussions. A.T. is funded by the European Union's Horizon 2020 research and innovation program under the Marie Sklodowska-Curie Grant Agreement No. 701647. Y.W. and A.J. are supported by startup funds at the University of Florida.
[1] C.-K. Chiu, J. C. Y. Teo, A. P. Schnyder, and S. Ryu, Rev. Mod. Phys. 88, 035005 (2016).

[2] S. Ryu, A. P. Schnyder, A. Furusaki, and A. W. W. Ludwig, New J. Phys. 12, 065010 (2010).

[3] A. Kitaev, in Advances in Theoretical Physics: Landau Memorial Conference, AIP Conference Proceedings, Volume 1134 (AIP, Melville, NY, 2009), p. 22.

[4] X. Chen, Z.-C. Gu, Z.-X. Liu, and X.-G. Wen, Phys. Rev. B 87, 155114 (2013).

[5] L. Fu, C. L. Kane, and E. J. Mele, Phys. Rev. Lett. 98, 106803 (2007).

[6] C. L. Kane and E. J. Mele, Phys. Rev. Lett. 95, 226801 (2005).

[7] C. L. Kane and E. J. Mele, Phys. Rev. Lett. 95, 146802 (2005).

[8] A. Y. Kitaev, Phys. Usp. 44, 131 (2001).

[9] F. Schindler, A. M. Cook, M. G. Vergniory, Z. Wang, S. S. Parkin, B. A. Bernevig, and T. Neupert, Sci. Adv. 4, eaat0346 (2018).

[10] W. A. Benalcazar, B. A. Bernevig, and T. L. Hughes, Science 357, 61 (2017).

[11] W. A. Benalcazar, B. A. Bernevig, and T. L. Hughes, Phys. Rev. B 96, 245115 (2017).

[12] Y. You, T. Devakul, F. Burnell, and T. Neupert, Phys. Rev. B 98, 235102 (2018).

[13] F. K. Kunst, G. van Miert, and E. J. Bergholtz, Phys. Rev. B 97, 241405(R) (2018).

[14] Z. Song, Z. Fang, and C. Fang, Phys. Rev. Lett. 119, 246402 (2017).

[15] M. Geier, L. Trifunovic, M. Hoskam, and P. W. Brouwer, Phys. Rev. B 97, 205135 (2018).

[16] Y. Wang, M. Lin, and T. L. Hughes, Phys. Rev. B 98, 165144 (2018).

[17] M. Ezawa, Phys. Rev. B 98, 045125 (2018).

[18] E. Khalaf, Phys. Rev. B 97, 205136 (2018).

[19] A. Matsugatani and H. Watanabe, Phys. Rev. B 98, 205129 (2018).

[20] M. Lin and T. L. Hughes, Phys. Rev. B 98, 241103(R) (2018).

[21] V. Dwivedi, C. Hickey, T. Eschmann, and S. Trebst, Phys. Rev. B 98, 054432 (2018).

[22] J. Langbehn, Y. Peng, L. Trifunovic, F. von Oppen, and P. W. Brouwer, Phys. Rev. Lett. 119, 246401 (2017).

[23] S. A. Parameswaran and Y. Wan, Physics 10, 132 (2017).

[24] Q. Wang, C.-C. Liu, Y.-M. Lu, and F. Zhang, Phys. Rev. Lett. 121, 186801 (2018).

[25] L. Trifunovic and P. W. Brouwer, Phys. Rev. X 9, 011012 (2019).

[26] A. Tiwari, M.-H. Li, B. A. Bernevig, T. Neupert, and S. A. Parameswaran, Phys. Rev. Lett. 124, 046801 (2020).
[27] T. Li, P. Zhu, W. A. Benalcazar, and T. L. Hughes, Phys. Rev. B 101, 115115 (2020).

[28] Y. You, arXiv:1908.04299.

[29] D. Călugăru, V. Juričić, and B. Roy, Phys. Rev. B 99, 041301(R) (2019).

[30] J. Ahn and B.-J. Yang, Phys. Rev. Res. 2, 012060 (2020).

[31] B. Roy, Phys. Rev. B 101, 220506 (2020).

[32] X. Wu, W. A. Benalcazar, Y. Li, R. Thomale, C.-X. Liu, and J. Hu, Phys. Rev. X 10, 041014 (2020).

[33] R.-X. Zhang, Y.-T. Hsu, and S. D. Sarma, Phys. Rev. B 102, 094503 (2020).

[34] R.-X. Zhang, J. D. Sau, and S. D. Sarma, arXiv:2003.02559.

[35] D. Vu, R.-X. Zhang, and S. D. Sarma, Phys. Rev. Res. 2, 043223 (2020).

[36] Z. Yan, F. Song, and Z. Wang, Phys. Rev. Lett. 121, 096803 (2018).

[37] Z. Yan, Phys. Rev. Lett. 123, 177001 (2019).

[38] E. Roberts, J. Behrends, and B. Béri, Phys. Rev. B 101, 155133 (2020).

[39] S. Ono, H. C. Po, and H. Watanabe, Sci. Adv. 6, eaaz8367 (2020).

[40] S. A. A. Ghorashi, X. Hu, T. L. Hughes, and E. Rossi, Phys. Rev. B 100, 020509(R) (2019).

[41] S. A. A. Ghorashi, T. L. Hughes, and E. Rossi, Phys. Rev. Lett. 125, 037001 (2020).

[42] E. Khalaf, W. A. Benalcazar, T. L. Hughes, and R. Queiroz, arXiv:1908.00011.

[43] N. Read and D. Green, Phys. Rev. B 61, 10267 (2000).

[44] D. A. Ivanov, Phys. Rev. Lett. 86, 268 (2001).

[45] D. M. Lee, Rev. Mod. Phys. 69, 645 (1997).

[46] C. Beenakker, Annu. Rev. Condens. Matter Phys. 4, 113 (2013).

[47] X.-L. Qi and S.-C. Zhang, Rev. Mod. Phys. 83, 1057 (2011).

[48] A. P. Schnyder, S. Ryu, A. Furusaki, and A. W. W. Ludwig, Phys. Rev. B 78, 195125 (2008).

[49] X.-L. Qi, T. L. Hughes, and S.-C. Zhang, Phys. Rev. B 81, 134508 (2010).

[50] Z. Wu, Z. Yan, and W. Huang, Phys. Rev. B 99, 020508(R) (2019).

[51] S. Ono, Y. Yanase, and H. Watanabe, Phys. Rev. Res. 1, 013012 (2019).

[52] A. Skurativska, T. Neupert, and M. H. Fischer, Phys. Rev. Res. 2, 013064 (2020).

[53] M. Geier, P. W. Brouwer, and L. Trifunovic, Phys. Rev. B 101, 245128 (2020).

[54] L. Fu, Phys. Rev. Lett. 106, 106802 (2011).

[55] E. Khalaf, H. C. Po, A. Vishwanath, and H. Watanabe, Phys. Rev. X 8, 031070 (2018). 
[56] R.-J. Slager, L. Rademaker, J. Zaanen, and L. Balents, Phys. Rev. B 92, 085126 (2015).

[57] J. Kruthoff, J. de Boer, J. van Wezel, C. L. Kane, and R.-J. Slager, Phys. Rev. X 7, 041069 (2017).

[58] J. Cano, B. Bradlyn, Z. Wang, L. Elcoro, M. G. Vergniory, C. Felser, M. I. Aroyo, and B. A. Bernevig, Phys. Rev. B 97, 035139 (2018).

[59] C. Y. Teo and C. L. Kane, Phys. Rev. B 82, 115120 (2010).

[60] F. Schindler, B. Bradlyn, M. H. Fischer, and T. Neupert, Phys. Rev. Lett. 124, 247001 (2020).

[61] C. Fang, M. J. Gilbert, and B. A. Bernevig, Phys. Rev. B 86, 115112 (2012).
[62] W. A. Benalcazar, T. Li, and T. L. Hughes, Phys. Rev. B 99, 245151 (2019).

[63] T. Fukui and T. Fujiwara, Phys. Rev. B 82, 184536 (2010).

[64] K. Shiozaki, T. Fukui, and S. Fujimoto, Phys. Rev. B 86, 125405 (2012).

[65] L. Fu and C. L. Kane, Phys. Rev. Lett. 100, 096407 (2008).

[66] X.-L. Qi, T. L. Hughes, and S.-C. Zhang, Phys. Rev. B 78, 195424 (2008).

[67] M. Stone and R. Roy, Phys. Rev. B 69, 184511 (2004).

[68] R. Jackiw and C. Rebbi, Phys. Rev. D 13, 3398 (1976).

[69] H. C. Po, L. Zou, T. Senthil, and A. Vishwanath, Phys. Rev. B 99, 195455 (2019). 